\title{
Influence of Acetaminophen on Pharmacokinetics and Pharmacodynamics of Midazolam in Healthy Volunteers
}

\author{
Dennis Feierman1,2, Tunga Suresh', Murali Pagala1, Jeffery Silverstein ${ }^{2}$ \\ ${ }^{1}$ Department of Anesthesiology, Maimonides Medical Center, Brooklyn, USA \\ ${ }^{2}$ Department of Anesthesiology, Mount Sinai School of Medicine, New York, USA \\ Email: dfeierman@maimonidesmed.org
}

Received 7 May 2015; accepted 9 June 2015; published 12 June 2015

Copyright (C) 2015 by authors and Scientific Research Publishing Inc.

This work is licensed under the Creative Commons Attribution International License (CC BY). http://creativecommons.org/licenses/by/4.0/

(c) (i) Open Access

\begin{abstract}
Background: Midazolam and acetaminophen are often co-administered in anesthesia practice. Both are metabolized by CYP 3A4 enzyme in the liver, and hence compete for the enzyme sites. This might lead to reduced metabolic breakdown and enhanced pharmacodynamic effects of midazolam in the presence of acetaminophen. Methods: The present study was undertaken to test this hypothesis. After IRB approval from Mount Sinai Medical Center, 15 healthy volunteers were used for 2 tests. For the first test, they were randomly assigned to receive oral doses of either midazolam $0.3 \mathrm{mg} / \mathrm{kg}$ in cherry syrup (Protocol A), or midazolam $0.3 \mathrm{mg} / \mathrm{kg}$ plus cherry flavored acetaminophen $15 \mathrm{mg} / \mathrm{kg}$ (Protocol B). At set intervals from 0 to $480 \mathrm{~min}$, the blood levels of midazolam and the subjects pulse rate, mean arterial pressure, respiratory rate, BIS index, and OAA/S scores were determined. After 2 weeks, the same subjects underwent the second test; they received the other medication protocol. Results: Acetaminophen slightly, but not significantly, increased the half life of blood midazolam, and the depressive effects of midazolam on the clinical signs of the subjects. Conclusion: These results lead us to conclude that there is no need to reduce the doses of midazolam when used in combination with acetaminophen.
\end{abstract}

\section{Keywords}

Midazolam, Acetaminophen, BIS Index, OAA/S Scores

\section{Introduction}

Midazolam is a frequently used benzodiazepine for inducing sedation in anesthesia. Oral midazolam is com-

How to cite this paper: Feierman, D., Suresh, T., Pagala, M. and Silverstein, J. (2015) Influence of Acetaminophen on Pharmacokinetics and Pharmacodynamics of Midazolam in Healthy Volunteers. Open Journal of Anesthesiology, 5, 116-121. http://dx.doi.org/10.4236/ojanes.2015.56022 
monly used preoperatively in pediatric patients. Oral administration of midazolam results in significant first-pass metabolism mediated by cytochrome P450 3A (CYP 3A4) enzyme in the liver [1]. Midazolam is often coadministered with acetaminophen, which has intrinsic analgesic properties. This combination raises an interesting pharmacological conundrum, as both are metabolized by CYP 3A4 enzyme in the liver [2] [3]. Since both midazolam and acetaminophen are substrates of CYP 3A4, and compete for its active site, using this combination could potentially affect the pharmacokinetic disposition and pharmacodynamic effects of midazolam due to a reduction in its first pass metabolism.

Our working hypothesis is that acetaminophen would inhibit CYP3A4 mediated metabolism of midazolam, leading to decreased metabolic clearance and increased bioavailability of midazolam. This could result in prolonged changes in the hemodynamic, respiratory, neuronal, and sedation indices in the patient receiving the combination of midazolam and acetaminophen. The primary aim of the present study was to determine the pharmacokinetic parameters of midazolam, such as maximal blood concentration $\left(\mathrm{C}_{\max }\right)$ and area under the curve (AUC), and its pharmacodynamic effects on hemodynamic, respiratory, neuronal, and sedation indices in healthy volunteers who receive oral midazolam with and without acetaminophen. The goal was to determine whether the dose of midazolam needed to be reduced when used in combination with acetaminophen, in order to avoid the possible clinical complications in the patients.

\section{Methods}

After obtaining the Institutional Review Board approval, this study was conducted at the General Clinical Research Center of the Mount Sinai School of Medicine over a 1 year period, and the data was analyzed at Maimonides Medical Center. This was a prospective double-blinded study involving sixteen healthy volunteers. Data from one patient was not included in the analysis because it was incomplete, as some of the blood samples where unobtainable.

After obtaining the informed consent, each volunteer underwent two tests that were conducted at least two weeks apart. For the first test, each volunteer was randomly assigned to receive oral doses of either midazolam $0.3 \mathrm{mg} / \mathrm{kg}$ in cherry syrup (Protocol A), or midazolam $0.3 \mathrm{mg} / \mathrm{kg}$ plus cherry flavored acetaminophen $15 \mathrm{mg} / \mathrm{kg}$ (Protocol B).

To determine the pharmacokinetic parameters, blood samples were collected from each volunteer at $0,15,30$, 45, 60, 90, 120, 180, 240 and 480 minutes after ingestion of the medications. Each blood sample was centrifuged and the plasma stored at $-40^{\circ} \mathrm{C}$ until analyzed. In each plasma sample, the level of midazolam was determined by the method of 4 using a Hewlett-Packard Model 5972 mass-selective detector (MSD). The $\mathrm{C}_{\max }$, AUC, and other parameters were determined using PK Solutions 2.0 pharmacokinetics program (Summit Research Services, Ashland, $\mathrm{OH}$ ).

The pharmacodynamic effects were assessed in each volunteer at $0,15,30,45,60,75,90,120,180,240$, and 480 minutes after ingestion of the medications, by monitoring the physiological parameters such as pulse rate, blood pressure, respiratory rate, $\mathrm{O} 2$ saturation, and Bispectral Index scores. We also collected the Observer Assessment of Alertness/Sedation (OAA/S) scores. The OAA/S score comprised of four components including responsiveness, speech, facial expression and eye evaluations. An independent investigator collected these data and was blinded with respect to the treatments.

The second test was carried out 2 weeks after the first test. Each volunteer acted as his/her own control, because for the second test each received the medications of either Protocol A or B that they were not given during the first test. In each, the pharmacokinetic and pharmacodynamic parameters were again determined as described above.

Mean ( \pm S.D.) values of each parameter at different time periods were calculated in the two treatment protocols. Between the two treatments, difference in each pharmacokinetic parameter was evaluated using paired ttest, and difference in the time-dependent changes in each pharmacodynamic parameter was evaluated using the repeated measures analysis of variance.

\section{Results}

The co-administration of acetaminophen and midazolam did not significantly affect the pharmacokinetic parameters of midazolam in the blood of tested volunteers (Table 1). $C_{\max }$ values were $247 \pm 258$ and $242 \pm 126$ 
Table 1. Pharmacokinetic parameters of serum midazolam in healthy volunteers following oral midazolam and midazolam plus acetaminophen.

\begin{tabular}{cccc}
\hline Pharmacokinetic parameter & Midazolam & Midazolam and acetaminophen & p value \\
\hline $\begin{array}{c}\text { Maximal concentration } \\
\left(\mathrm{C}_{\max }\right)(\mathrm{ng} / \mathrm{ml})\end{array}$ & $247 \pm 258$ & $242 \pm 126$ & 0.45 \\
$\begin{array}{c}\text { Time to maximal concentration } \\
\left(\mathrm{T}_{\max }\right)(\mathrm{min})\end{array}$ & $92 \pm 31$ & $91 \pm 31$ & 0.44 \\
$\begin{array}{c}\text { Time to half decline in concentration } \\
\left(\mathrm{t}_{1 / 2}\right)(\mathrm{min})\end{array}$ & $166 \pm 60$ & $195 \pm 63$ & 0.07 \\
$\begin{array}{c}\text { Area under the curve (AUC) } \\
(\mathrm{g} \text {-min/ml) }\end{array}$ & $93 \pm 99$ & $85 \pm 64$ & 0.24 \\
$\begin{array}{c}\text { Rate of clearance } \\
(\mathrm{ml} / \mathrm{min} / \mathrm{kg})\end{array}$ & $4.8 \pm 2.1$ & $4.5 \pm 1.7$ & 0.67 \\
\hline
\end{tabular}

Values are Mean \pm S.D. obtained from 15 subjects who received the two sets of medications 2 weeks apart. Difference in the values of each parameter in the two groups has been evaluated using paired t-test.

$\mathrm{ng} / \mathrm{ml}(\mathrm{p}=0.45)$ following treatment protocols A and B respectively. The AUC values were $93 \pm 99$ and $85 \pm 64$ ug-min $/ \mathrm{ml}(\mathrm{p}=0.24)$ following A and B protocols respectively. Similarly, the time to maximal concentration $\left(\mathrm{T}_{\max }\right)$, the time to half decline in concentration $\left(\mathrm{t}_{1 / 2}\right)$ and the rate of clearance (CL) did not differ significantly following the two treatment protocol (Table 1).

A similar trend as above was observed in most of the pharmacodynamic parameters following the two treatment protocols. In volunteers who received midazolam alone, the pulse rate was $74 \pm 16$ pulses/min initially, and it gradually decreased to $67 \pm 12 \mathrm{pulses} / \mathrm{min}$ by $120 \mathrm{~min}$, but went back to the initial value by $480 \mathrm{~min}$ (Figure 1). In the same volunteers who received midazolam plus acetaminophen, the pulse rate was $70 \pm 14$ pulses/min initially, and it showed about the same degree of decrease and subsequent increase as in volunteers who received midazolam alone (Figure 1). There was no statistically significant difference in the time dependent changes in the pulse rate between the two groups, probably refecting the minimal effects, at the dose tested, that acetaminophen has on the disposition of midazolam (Table 1).

In volunteers who received midazolam alone, the mean arterial pressure was $88 \pm 10 \mathrm{mmHg}$ initially, and it gradually decreased to $71 \pm 11 \mathrm{mmHg}$ by $120 \mathrm{~min}$, but went back to $81 \mathrm{mmHg}$ by $480 \mathrm{~min}$ (Figure 2). In the same volunteers who received midazolam plus acetaminophen, the mean arterial pressure was $86 \pm 8 \mathrm{mmHg}$ initially, and it showed about the same decrease, but lower subsequent increase as in volunteers who received midazolam alone (Figure 2). There was no statistically significant difference in the time dependent changes in the mean arterial pressure between the two groups.

In subjects who received midazolam alone, the respiratory rate was $19 \pm 1$ breaths/min initially, and it did not much change over 480 (Figure 3). In the same subjects who received midazolam plus acetaminophen, the respiratory rate was the same as in the first group, but gradually reduced to $16 \pm 3$ breaths/min by $120 \mathrm{~min}$, and it slowly increased to 18 beats/min by $480 \mathrm{~min}$ (Figure 3). The respiratory rate after $480 \mathrm{~min}$ was significantly lower $(p=0.025)$ in subjects who received midazolam plus acetaminophen.

Subjects who received midazolam alone, BIS index was $96 \pm 3$ units initially, and it showed a time dependent polyphasic change, decreasing to $77 \pm 16$ units by $60 \mathrm{~min}$, then slightly increasing, again decreasing to $73 \pm 19$ by $180 \mathrm{~min}$, and finally reaching 90 by $240 \mathrm{~min}$ (Figure 4). In the same subjects who received midazolam plus acetaminophen, the BIS index showed about the same changes as in subjects who received midazolam alone (Figure 4). There was no statistically significant difference in the time dependent changes in the BIS index between the two groups.

Similarly, subjects who received midazolam alone, the total OAA/S score was $20 \pm 0$ units initially, and it also showed a time dependent polyphasic change, decreasing to $14 \pm 5$ units by $45 \mathrm{~min}$, then slightly increasing, again decreasing to 14 by $120 \mathrm{~min}$, and finally reaching 20 by $480 \mathrm{~min}$ (Figure 5 ). In the same subjects who received midazolam plus acetaminophen, the total OAA/S score showed about the same changes as in subjects who received midazolam alone (Figure 5). There was no statistically significant difference in the time dependent changes in the OAA/S score between the two groups. 


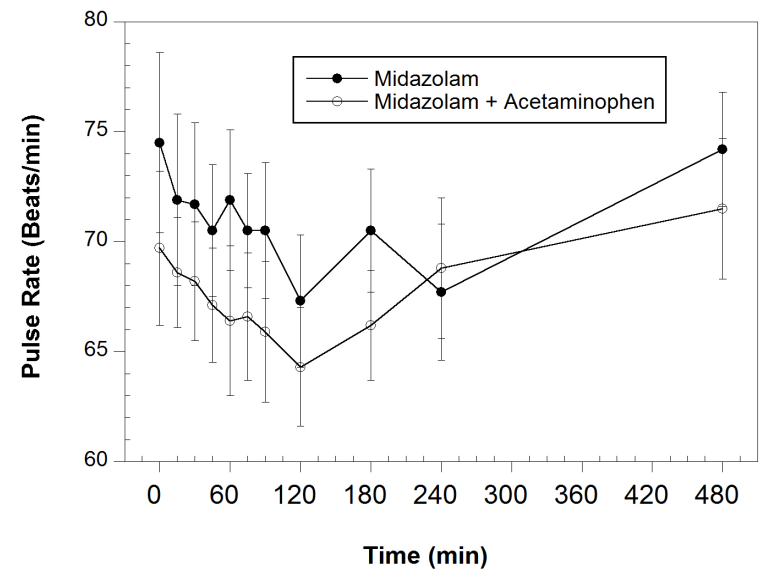

Figure 1. Pharmacodynamic effects of midazolam in the presence and absence of acetaminophen on the pulse rate in healthy human volunteers. Each circle in the graph represents the mean value, and the vertical line represents the standard deviation obtained from 15 subjects. Repeated measures ANOVA showed no significant difference the values of the two groups.

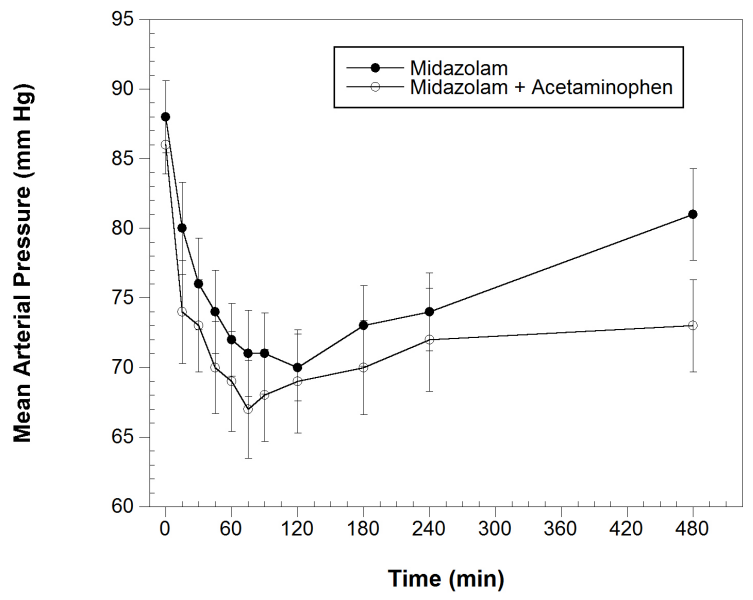

Figure 2. Pharmacodynamic effects of midazolam in the presence and absence of acetaminophen on the mean arterial pressure in healthy human volunteers. Other details are the same as Figure 1.

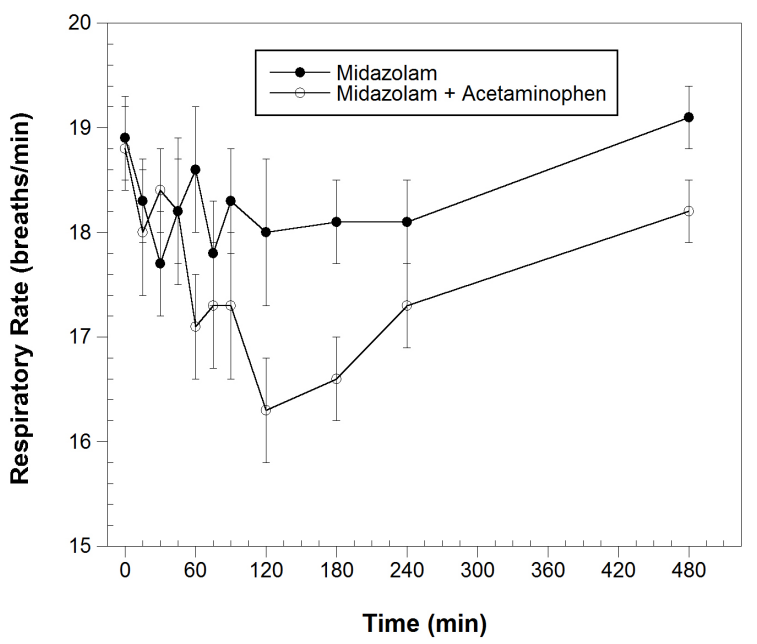

Figure 3. Pharmacodynamic effects of midazolam in the presence and absence of acetaminophen on the respiratory rate in healthy human volunteers. Other details are the same as Figure 1. 


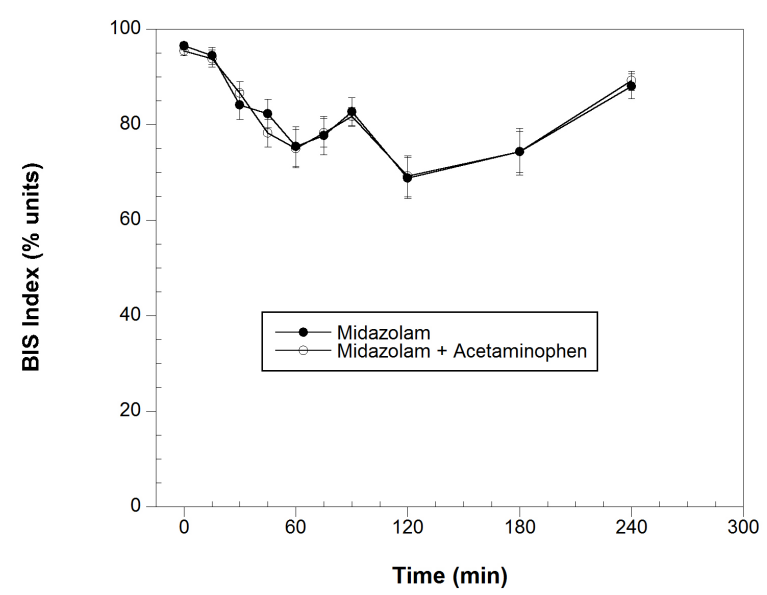

Figure 4. Pharmacodynamic effects of midazolam in the presence and absence of acetaminophen on the respiratory rate in healthy human volunteers. Other details are the same as Figure 1.

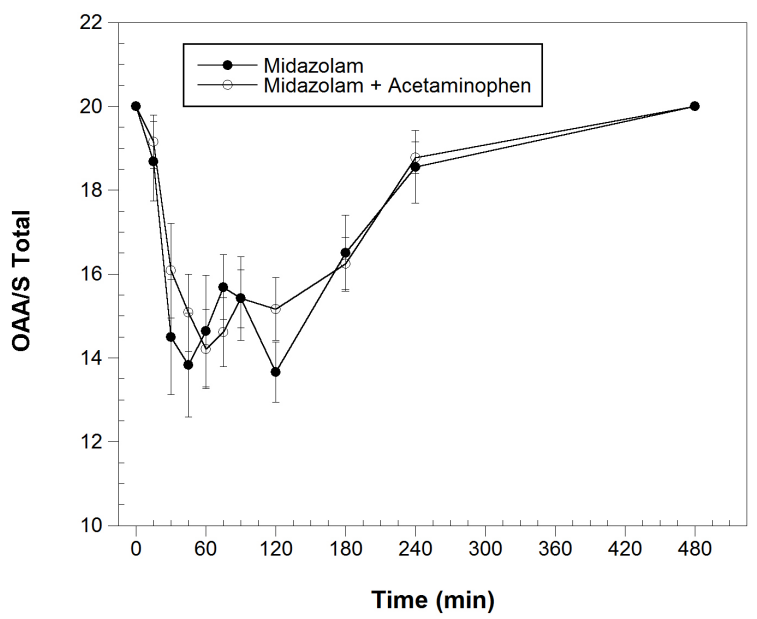

Figure 5. Pharmacodynamic effects of midazolam in the presence and absence of acetaminophen on total OAA/S score in healthy human volunteers. Other details are the same as Figure 1.

\section{Conclusion}

These studies showed that midazolam by itself caused a transient reduction in the pulse rate, mean arterial pressure, respiratory rate, BIS index, and the total OAA/S score for the first 60 to $120 \mathrm{~min}$, and then these parameters reached their initial values in the next 120 min (Figures 1-5). Acetaminophen slightly enhanced these initial depressive effects of midazolam (Figures 1-5). Although these depressive effects of acetaminophen are not statistically significant, they are consistent with slightly increased half-life of midazolam (Table 1), possibly due to reduced metabolic breakdown of midazolam by P450 3A in the presence of acetaminophen. These results are consistent with the interaction of midazolam and acetaminophen for the P450 3A enzyme sites [2] [3].

Our results showed that acetaminophen had little effect on $\mathrm{C}_{\max }$ and AUC of midazolam and therefore it appeared that it had little effect on absorption or bioavailability. Although both midazolam and acetaminophen are metabolized by the same P450, acetaminophen may have a high Ki for the inhibition of midazolam metabolism, as it was reported to have a $\mathrm{Ki}$ of $3 \mathrm{mM}$ for fentanyl metabolism [4]. It appears that at the dose tested, acetaminophen has little clinical effect on the disposition of midazolam. Based on these considerations, it may be concluded that there is no need to reduce the dose of midazolam when used in combination with acetaminophen.

\section{References}

[1] Gorski, J.C., Jones, D.R., Haehner-Daniels, B.D., Hamman, M.A., O’Mara Jr., E.M. and Hall, S.D. (1998) The Con- 
tribution of Intestinal and Hepatic CYP3A to the Interaction between Midazolam and Clarithromycin. Clinical Pharmacology and Therapeutics, 64, 133-143. http://dx.doi.org/10.1016/S0009-9236(98)90146-1

[2] Kronbach, T., Mathys, D., Umeno, M., Gonzalez, F.J. and Meyer, U.A. (1989) Oxidation of Midazolam and Triazolam by Human Liver Cytochrome P450IIIA4. Molecular Pharmacology, 36, 89-96.

[3] Kostrubsky, V.E., Szakacs, J.G., Jeffery, E.H., Wood, S.G., Bement, W.J., Wrighton, S.A., Sinclair, P.R. and Sinclair, J.F. (1997) Role of CYP3A in Ethanol-Mediated Increases in Acetaminophen Hepatotoxicity. Toxicology and Applied Pharmacology, 143, 315-323. http://dx.doi.org/10.1006/taap.1996.8081

[4] Feierman, D.E. (2000) The Effect of Paracetamol (Acetaminophen) on Fentanyl Metabolism in Vitro. Acta Anaesthesiologica Scandinavica, 44, 560-563. http://dx.doi.org/10.1034/j.1399-6576.2000.00513.x 